\title{
Alliances militaires et musicales sur le mont Nyiro: le système d'âge des Samburu à l'épreuve des nouveaux paradigmes du conflit
}

\section{Giordano Marmone}

\author{
(2) OpenEdition \\ Journals \\ Édition électronique \\ URL : https://journals.openedition.org/eastafrica/349 \\ DOI : $10.4000 /$ eastafrica.349 \\ ISSN : 2790-1076 \\ Éditeur \\ IFRA - Institut Français de Recherche en Afrique
}

Édition imprimée

Date de publication : 1 septembre 2014

Pagination : 87-98

ISSN : 2071-7245

Référence électronique

Giordano Marmone, "Alliances militaires et musicales sur le mont Nyiro: le système d'âge des Samburu à l'épreuve des nouveaux paradigmes du conflit », Les Cahiers d'Afrique de l'Est / The East African Review [En ligne], 49 | 2014, mis en ligne le 07 mai 2019, consulté le 09 décembre 2021. URL: http://journals.openedition.org/eastafrica/349; DOI : https://doi.org/10.4000/eastafrica.349 


\title{
Alliances militaires et musicales sur le mont Nyiro: le système d'âge des Samburu à l'épreuve des nouveaux paradigmes du conflit
}

\author{
Giordano Marmone ${ }^{3}$
}

\section{Introduction}

Chez les Samburu du nord Kenya, la production musicale des deux dernières décennies et les nouvelles formes de participation aux danses sont un témoin privilégié des transformations subites et radicales qui ont affecté ces pasteurs du Kenya centreseptentrional. L'apparition de la kalachnikov, de G-3 et de M-16, en provenance des zones en crise de la Corne de l'Afrique, a entraîné un changement dans les modalités de défense du territoire et dans l'organisation des vols de bétail. Anciens, guerriers et membres des communautés proches ont été obligés de créer des alliances stables pour faire face à la capacité de résistance et d'attaque des populations victimes de leurs raids, accrue par l'usage des armes à feu. Ces nouvelles formes d'alliance - intergénérationnelle et territoriale - ont changé si profondément les paradigmes d'interaction entre les individus, les membres des différentes communautés samburu et les groupes d'âge, que même leur culture et leur pratique musicales ont évolué en s'adaptant de façon surprenante aux changements en cours dans la société. La création d'une série d' " alliances musicales »a constitué un des résultats les plus surprenants de ce processus.

\section{Armes à feu en pays Samburu}

L'introduction massive des armes à feu dans le district Samburu à partir de 1995, en provenance des zones en crise de la Corne de l'Afrique (Mkutu, 2008), a altéré radicalement les règles du jeu dans la longue histoire de batailles et de vols de bétail réciproques qui mettent aux prises Turkana et Samburu, peuples rivaux de pasteurs tous deux d'origine nilotique. L'utilisation durable de fusils automatiques comme la kalachnikov et le G-3 au cours des razzias a profondément changé les modalités de la pratique traditionnelle, multiséculaire, des raids conduits jusqu'à il y a vingtaine d'années avec l'usage presque exclusif - au moins du côté des Samburu - des lances et des flèches.

3 Doctorant, membre du centre CREM d'ethnomusicologie du LESC (université Paris Ouest UMR 7186). 
Les effets les plus violents de cette nouveauté en matière militaire ont été observés dans le nord du district Samburu - devenu county en 2012 - notamment dans le territoire proche du mont Nyiro où la présence de nombreuses enclaves Turkana en territoire Samburu (comme les villages de Nasura et de Kawap), ou encore de petites villes au sein desquelles les deux communautés résident côte à côte (Baragoi), avait depuis longtemps fait de cette région le lieu privilégié pour l'organisation et la mise en œuvre de raids par les moran (guerriers) des deux tribus.

Les armes à feu sont loin d'être la cause première des tensions entre Turkana et Samburu, mais en transformant la pratique de vol de bétail qui s'apparente de plus en plus à des épisodes de guérilla pouvant causer nombre de morts tant chez les guerriers que chez les femmes et les enfants, leur présence et leur usage prolongés ont créé dans cet espace des conditions d"insécurité et de peur constantes qui, dans le cas des Samburu, ont eu des répercussions importantes au sein de la société.

\section{« En raid avec mon père "}

Dans la partie septentrionale de la région, un des résultats le plus notable de cette situation de « chronic, low-intensity warfare » (Pike, Straight et al. : 2009) consiste en une différenciation de plus en plus marquée entre d'un côté, les communautés samburu du sud et du centre, très rarement ou presque jamais touchées par ces épisodes de violence, et de l'autre celles du nord où la menace constante d'agression avec des armes à feu a obligé les communautés impliquées à se réunir dans de vrais villages semi-permanents. Les rassemblements de ce type n'existaient pas dans le dispositif territorial des Samburu : ils ont été créés, presque exclusivement au nord, à des fins explicites d'auto-défense, les établissements isolés étant devenus des cibles trop faciles pour les attaquants. Guerriers et anciens ont ainsi commencé à travailler de concert pour protéger leurs familles et leurs troupeaux, de façon à pouvoir aligner un nombre suffisant d'hommes prêts à se battre en cas de razzia, alors même que dans un passé très récent les fonctions de protection et de combat étaient la prérogative presque exclusive des moran.

La société des Samburu se fonde sur une structure dite "à statuts d'âge » (moran guerriers / anciens, aînés) dans laquelle, aux séparations générationnelles entre jeunes et aînés correspond une répartition tout aussi nette des obligations sociales et des tâches dans le domaine de l'organisation du travail. Ces divisions structurelles (lesquelles de toute façon sont en train de se défaire partout chez les Samburu pour des motifs qu'il serait hors sujet d'exposer ici) se sont notablement affaiblies dans les communautés du mont Nyiro.

Aux cours de ces dernières années, l'obligation de créer des groupes de feu suffisamment puissants pour contrecarrer les assauts des raids ennemis, a poussé les anciens à changer partiellement leur style de vie : ils continuent à porter des armes au-delà de la période requise de lmurrano (pendant laquelle durant 15 ans tous les hommes doivent 
en quelque sorte faire leur service militaire en tant que moran) et, autre changement, les anciens continuent à mener les raids guerriers non seulement dans les moments de défense du territoire, mais aussi à l'occasion des raids organisés pour récupérer les têtes de bétail volées.

La participation des anciens aux raids des moran ne constitue pas à proprement parler une nouveauté absolue ; elle semble en effet avoir été à l'œuvre au moins depuis les années 1960. Le vrai élément de discontinuité avec le passé, outre l'introduction des fusils, est représenté par la dimension de cette participation. Tous les témoignages recueillis sur le terrain témoignent d'un changement radical et établissent le lien entre l'intensité du phénomène et la diffusion massive, il y a vingt ans, des fusils d'assaut. Un jeune ancien, guerrier dans les années 1990, nous l'a expliqué de façon très claire : «Dans le passé [les anciens qui] allaient [en raid avec nous guerriers] étaient très peu nombreux, alors qu'aujourd'hui les anciens ont les fusils, les moran ont les fusils, et tout le monde y va [en raid] » (« Kopuo apa lobo obo, kore taata keata lpayani ntioli, keata lmurran ntioli, kopuo pooki »). Un autre ancien, guerrier dans les années 1960-1970, nous a raconté que les anciens qui allaient en raid avec les moran étaient « Un, parfois deux. Les Lkimaniki [nom de la génération des jeunes anciens de l'époque, NDA] ne connaissaient pas la bataille parce que à ce moment-là il y avait l'armée des blancs » (« Lobo, oare. Meyolo Lkimaniki larrabal amu keti apa sirkali ee ltun'gana oibor"). ${ }^{4}$

Dans les dernières années, la situation a changé de manière radicale. Les luttes au corps à corps ou à la lance, qui requéraient une dose considérable de force physique et étaient pour cette raison réservées aux moran, ont été presque définitivement abandonnées. Avec l'appui d'une kalachnikov, les anciens seniors peuvent désormais continuer de participer aux actions les plus effectives, en se révélant parfois même des combattants meilleurs que les guerriers, car ils ont développé au fil du temps une connaissance plus approfondie du maniement des fusils. Un jeune maître d'école primaire d'un village situé aux flancs du mont Nyiro nous a révélé, à propos d'un grand raid effectué par les Samburu dans la zone de Nasura contre les Turkana en avril 2012 : « Si tu étais venu ici le jour de Nasura, tu n'aurais trouvé que les femmes, les enfants et Mara Narok [1'homme le plus ancien du village, NDA]. Tous les hommes de ce côté du mont Nyiro étaient partis en raid à Nasura. Personne n'était resté ici ».

4 Il se réfère ici à la période coloniale durant laquelle le contrôle exercé par les Anglais sur les activités des Samburu, jusqu'en 1963 - date de l'indépendance - avait réduit drastiquement la pratique des raids. 


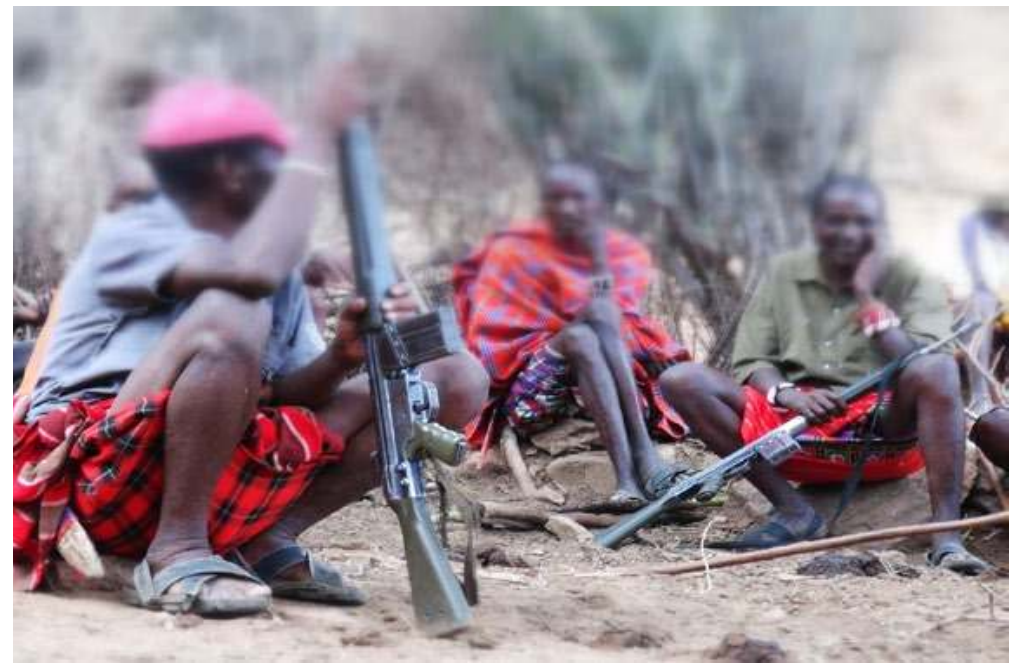

Anciens continuant de combattre avec des fusils (Photo: Marmone 2013)

\section{Combattre ensemble, danser ensemble}

Une modification dans les dynamiques internes à la société samburu et dans les rôles respectifs endossés par les membres des différents statuts d'âge et des générations ne peut qu'altérer profondément la conception que chaque individu a de son positionnement au sein de la communauté. L'effacement (relatif tout de même) des frontières générationnelles est à la base d'une re-négociation des modèles d'interaction entre jeunes et aînés et d'une transformation conséquente des pratiques coutumières.

Ainsi le patrimoine musical - reflet mais aussi théâtre producteur de la structure sociale des Samburu, avec ses différents répertoires, un pour chaque génération, et ses « chants de statut d'âge » au travers desquels enfants, moran et anciens transforment en mélodie chorale le récit de leur identité d'hommes en transformation - a connu, dans les deux dernières décennies et surtout au nord du county, une évolution inattendue mais intrinsèquement liée aux évènements décrits ci-dessus.

Chez les Samburu, non seulement les répertoires, mais aussi la pratique musicale et la participation aux danses constituent une représentation vivante et explicite des « coupures »-comme les appelle Straight (2005) - qui séparent les groupes d'hommes d'âge et partant de rôles différents. Pour donner un exemple, un observateur attentif resterait sûrement frappé en assistant à la traditionnelle double exécution des danses cérémonielles de l'après-midi, pratique commune à tout le pays Samburu (à l'exception du nord, comme nous allons voir), déjà observée et décrite par Paul Spencer pendant son terrain conduit 
au début des années $1960^{1}$ : moran et anciens, tout en mobilisant dans ce cas la même trame de chants et de danses, préfèrent se séparer en créant deux groupes de danseurs différenciés par l'appartenance à un des statuts d'âge. Les anciens dansent d'abord avec les femmes mariées et, après seulement et en se déplaçant dans un lieu différent, les moran s'y emploient à leur tour et répètent les mêmes danses, cette fois-ci accompagnés par les filles.

La répétition de la même trame musicale cérémonielle, en deux moments et dans deux lieux différents, a l'effet de rendre encore plus manifeste l'écart générationnel entre les deux groupes d'âge. En « sur-jouant» leur incompatibilité statutaire au niveau musical, anciens et guerriers donnent à voir, à percevoir et reproduisent la distance sociale qui les sépare.

Chez les communautés du nord et en particulier celles du mont Nyiro, la pratique de la double exécution des chants et des danses cérémonielles que nous venons de décrire, n'a aujourd'hui plus cours. La session musicale, devenue unique, réunit anciens et guerriers qui dansent et chantent ensemble, au même moment et dans le même espace. Même les solistes qui se succèdent à la tête du groupe des chanteurs-danseurs appartiennent indistinctement aux deux statuts d'âge. L'expression musicale et chorégraphique des « coupures » générationnelles, si évidentes dans les communautés samburu du sud et du centre, semble disparaître et perdre de sa force symbolique dans la partie septentrionale du county.

Les anciens et les guerriers s'accordent pour l'affirmer que la disparition de la « double exécution des danses cérémonielles », et par conséquent l'expression la plus explicite de la distance musicale entre les deux statuts d'âge, s'est produite au cours des dix-quinze dernières années. "Quand j'étais moran les anciens répétaient les danses » nous a expliqué un jeune ancien, guerrier dans les années 1990. « Nous, les Lmetili, [nom de la génération des moran actuels, NDA] avons été les premiers à danser avec tous les anciens » a ajouté un guerrier, présent pendant la même conversation.

Arrivé à ce point, il faut se poser la question. Pourquoi y-a-t-il une différence si nette dans la pratique musicale au sein de la même population ? Les réponses obtenues à ce propos sur le terrain ne laissent pas de place à la tergiversation. S.L., chef des moran d'un village proche du mont Niyro et soliste parmi les plus appréciés de la zone, à la question « Pourquoi ici anciens et guerriers dansent-ils ensemble, alors qu'ailleurs, au moment de chanter, ils se séparent? »répond :

[Parce-que] nous on s'aime à cause de la peur. [...] Tu sais, dans ces endroits-là [le sud et le centre du county, NDA] les moran et les anciens se détestent [parce qu'] il n'y a pas la

1 «In the afternoon this [une cérémonie de circoncision, NDA] gives way to a general dance in which the elders may dance with the wives; and, later, the moran with the girls » (Spencer1973: 88). 
peur («Ikishamai tangarake nguriesho. [...] Iyolo, inina kop naibaro lmarn o lpayani meatai nguriesho »).

Les témoignages recueillis au sein des communautés du mont Niyro établissent un lien très net entre l'apparition des armes à feu, source de violence et d'insécurité, et l'altération des structures institutionnelles traditionnelles. Ces changements, comme on a pu le voir, ont eu des conséquences évidentes sur la pratique musicale des Samburu du nord.

L'alliance militaire à laquelle moran et anciens ont été contraints pour faire face au péril constant des attaques ennemies, de plus en plus fréquentes dans les dernières années, a eu un résultat inattendu et, d'une certaine manière, surprenant : une alliance musicale, reflet et conséquence des nouveaux schémas de coopération entre les membres des deux statuts d'âge.

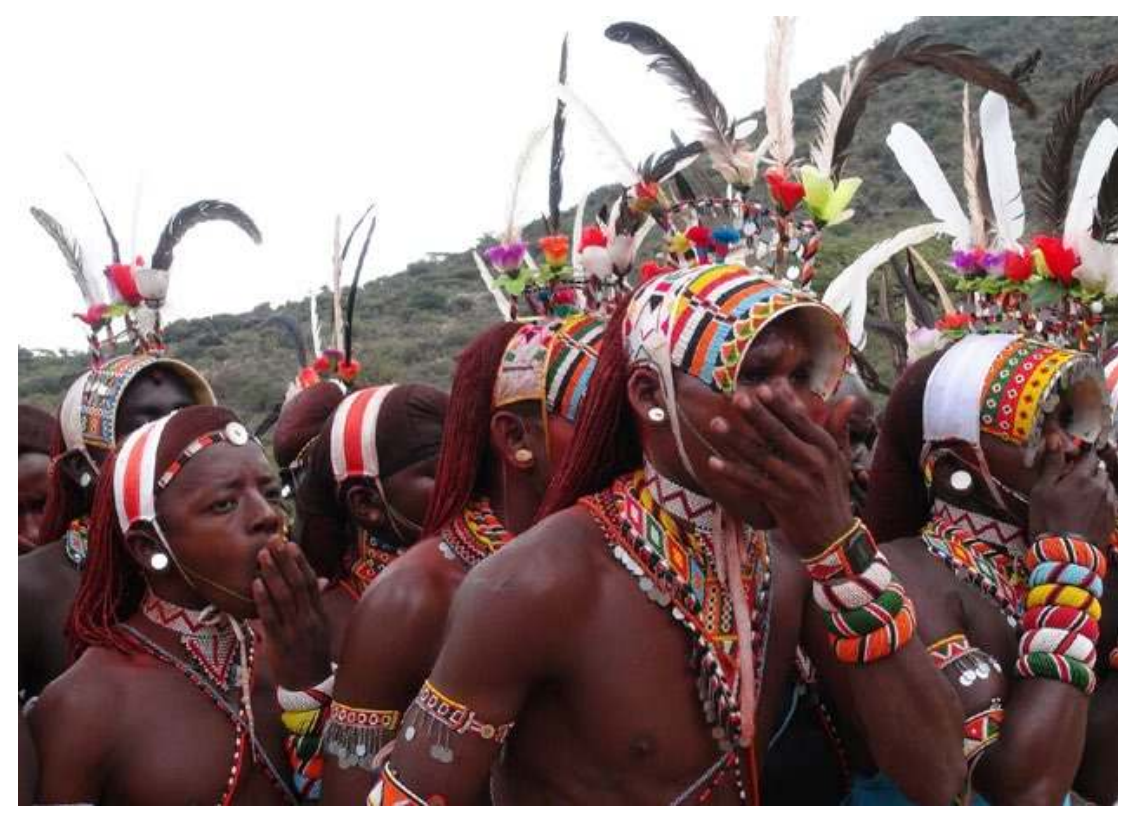

Danses et chants de guerriers (Marmone 2013)

\section{Un « territoire musical » partagé}

Mais l'action de défense du bétail et de la communauté ne peut pas s'appuyer uniquement sur la collaboration intergénérationnelle. Il faut, en même temps, créer une alliance territoriale entre guerriers et anciens des villages proches, propre à amplifier l'impact d'une action (ou d'une réaction) militaire. 
Chez les Samburu, chaque village ou tout établissement dispose d'un groupe de guerriers, nés et grandis ensemble, destinés à défendre leur territoire d'origine et ses habitants. Ces groupes (appelés « clubs » par Spencer (1965), et surnommés par les guerriers «keai (sg. kea), de K.A. acronyme de Kenyan Army) sont caractérisés par une structure de commandement qui prévoit la présence d'un ou plusieurs chefs (normalement un maximum de trois ou quatre) appelés Larikok (sg. Larikoni), qui sont aussi les représentants des anciens chez les guerriers. En absence de conflits et de tensions particulières, les rapports entre les différents groupes se fondent surtout sur des collaborations de travail occasionnelles liées aux tâches de l'élevage du bétail et sur la coparticipation aux cérémonies et aux danses cérémonielles organisées dans les territoires proches.

Dans le domaine musical, chaque groupe peut compter sur la présence de plusieurs solistes dont l'autorité est reconnue au sein de ses membres. Il n'est pas rare, cependant, que cette autorité musicale trouve à se mesurer à celle des solistes des autres keai. Dans le sud et le centre du county Samburu, si un groupe de moran décide de se déplacer dans un village proche pour participer à une cérémonie, et donc aux danses et aux chants, les solistes dudit groupe se trouveront en position subalterne par rapport aux solistes du groupe local : ils disposeront d'un temps limité pour chanter, à l'inverse des solistes et des membres de la kea locale qui occuperont l'essentiel du temps.

Chez les moran du nord, on constate au niveau social et musical des dynamiques sensiblement différentes. Les groupes de guerriers du nord sont souvent unis par des alliances territoriales plus solides que celles établies ailleurs dans le territoire Samburu. Ces alliances ont été scellées, rappelons-le, pour des raisons d'auto-défense et rendre plus efficaces leurs actions d'attaque et leurs razzias contre les villages turkana. Les décisions les plus importantes sont prises de façon collégiale, lors de grandes assemblées qui incluent les moran, tous les larikok des keai alliés et les plus influents anciens des villages impliqués.

On va le voir, le réaménagement des solidarités consécutives à l'insécurité a eu ici un autre impact, complémentaire du précédent, non pas seulement vertical au sein de la hiérarchie sociale des âges, mais aussi horizontal, au sein des membres de même statut d'âge, et tout aussi conséquent sur la pratique musicale et ses arrangements structurels caractéristiques.

L'idée de « territoire » chez les moran (et les anciens) du mont Nyiro, a profondément changé en réponse à l'augmentation exponentielle des cas de razzia aux armes à feu à partir des années 1990. Le lieu d'origine est encore reconnu comme le lieu d'appartenance du groupe des guerriers (la kea de Waso Rongai, la kea de Larerok, la kea de Tuum, etc.), mais l'« esprit de clocher », par ailleurs si caractéristique et qui porte les guerriers à s'identifier presque exclusivement à leur lieu de naissance, est ici presque totalement absent. Les 
guerriers des villages du mont Nyiro aiment se définir, en fait, comme «lmurran le manete Nyiro », « les moran de ce côté du Nyiro ». La nécessité d'une alliance contre les Turkana a peu à peu créé un lien entre ces communautés, soumises aux mêmes difficultés et à la même sensation d'insécurité, entraînant une modification du sentiment d'appartenance et de la conception territoriale.

De quelle façon cela se reflète-t-il dans la pratique musicale ? On a déjà dit que chez les Samburu chaque groupe de guerriers dispose de plusieurs solistes (jusqu'à cinq à six, parmi lesquels un ou deux sont considérés comme les plus doués et partant les plus importants) dont l'autorité musicale est fortement conditionnée par le lien solide qui les rattache à la zone ou à leur village d'origine.

L'aire d'influence des solistes des keai du nord est, au contraire, extrêmement plus vaste et s'étend à tous les territoires des groupes alliés. Cela implique que, si le groupe de Tuum doit se trouver à Larerok pour participer à une cérémonie, ses solistes ont le droit de diriger les danses en chantant autant voire parfois davantage que les solistes locaux. Ce genre de dynamique, très commune au nord, est au contraire difficilement observable dans le reste du pays samburu.

Chez les moran samburu, les solistes revêtent le rôle fondamental de représentants, dans le domaine musical, de toute la kea : le poids de leur autorité est, d'une certaine façon, directement proportionnel à l'importance du groupe dans un territoire déterminé. L'alliance militaire entre les keai du Nyiro a fortifié leur rapport de collaboration et leurs interactions jusqu'à un niveau presque inconnu dans le reste du county et cette affinité, entretenue par une condition de péril permanent qui a amené à une nouvelle forme de collaboration entre groupes, a contribué à l'affaiblissement des sentiments d'appartenance locale. Un tel changement de perspective, comme on a vu, a eu des conséquences directes sur la pratique musicale : les solistes des différentes keai du Nyiro, tout en gardant leur statut et leur prestige social, ont perdu, paradoxalement, au moins en partie, leur pouvoir dans le lieu d'origine du groupe auquel ils appartiennent. Dans le même temps, ils ont gagné le droit d'exercer leur autorité dans une zone extrêmement plus ample. Comme dans la configuration précédente, une alliance militaire est à l'origine d'une forme inédite de convergence au niveau musical.

\section{De la lance au fusil : la révolution dans les textes chantés}

Le passage soudain des lances aux fusils d'assaut, réalisé chez les communautés du nord dans un laps de temps de dix à quinze ans seulement, a eu des répercussions manifestes au sein de la culture musicale des Samburu. Nous avons apprécié les profondes modifications dans les modalités de participation aux danses et aux chants collectifs, consécutives à l'insécurité et à l'état de guerre permanent à basse intensité caractéristique de cette région frontière. 
De la même façon, les armes à feu sont devenues un véritable objet de culte pour les moran samburu. Dans le nord du territoire, chaque guerrier doit obligatoirement se procurer un fusil, pour le prix de cinq ou six vaches. Les lances « résistent » encore entre les mains de quelques anciens nostalgiques, mais les jeunes les considèrent aujourd'hui comme des objets sans aucune utilité 2 . Cet élément témoigne d'une progressive et rapide perte de prestige et d'intérêt militaire de ce qui, encore dans un passé récent, étais le symbole par excellence de l'état du guerrier.

Cette transition cruciale a laissé des traces visibles au sein du corpus des chansons et des textes chantés composés par les moran Samburu au cours des derniers vingt ans.

Comparons un chant (I) créé par la génération Lmooli dont les membres, aujourd'hui jeunes anciens, étaient guerriers pendant les années quatre-vingt-dix, avec les textes extraits de deux chants (IIa, b) composés par les membres de la génération Lmetili, les guerriers actuels

\section{(I) Lwampa lan'g soro mperria}

Loidikò le yeyo, otumo lkaria

Kutoporiè ldarancha marti

Soro mperria e lmurran

Kedorropi sesen e ntoye

Oye enne e lkaria

Lwampa lan'g oiroshi mperria

Nongusià lai, tejo sèere

Oye enne e lkaria

Lwampa lan'g arrama mperria

Notre groupe de raiders aux lances violettes [oxydées]

Cher ami, prends l'ocre rouge

On a traversé un pont dans le lieu aux pierres noires

Les lances des moran sont violettes

Et le corps des filles est petit

Dans ce lieu d'ocre rouge

Les lances de notre groupe de raiders sont lourdes

Ma mère, dis « Salut! »

Dans ce lieu d'ocre rouge

[Se trouvent] Les nombreuses lances de notre groupe de raiders

2 Ils les appellent ironiquement lorisié, comme le petit couteau utilisé par les femmes pour le façonnage du cuir. 
II) a

Nanyore e L... [nom du guerrier]

Naitoipoye lmurran

Kitidirie nairimuo

To lpurkelli ltaleta

Kijo mutut kalben

Narimuo torua lchomwa

Le [fusil] vert de L... ${ }^{3}$

Protège les guerriers

On a escaladé [le mont] avec celui aux trous ${ }^{4}$

Dans la plaine aux collines

Nous disons qu'il faut que tu aies une carabine

Celui aux trous expulse les cartouches

b)

...

Tonin'go G-3 eyogoki larrabal

Naaku nterito kukurto ta Lan'gatuny

Nearash Namalit ngorii e nkuma

Nodoru nambai e nkuma e kalashnikov

Écoute [comme] le G-3 a mugi dans la bataille

La poudre commença [à se lever] lorsque on combattait à Lan'gatuny

Le son [des coups] de feu laissa des sillons à Namalit

Les nombres [incisés] sur les kalachnikovs devinrent rouges de feu

En analysant les textes composés par ces deux générations de guerriers, celle des années 1990 et celle des années 2000, on a le sentiment de se trouver en présence de chants provenant de deux époques bien différentes, très éloignées l'une de l'autre. Un changement si rapide et profond ne peut être expliqué qu'au travers du choc consécutif à l'introduction massive des armes à feu chez les communautés du nord Samburu. Le fusil d'assaut est ainsi devenu non seulement le moyen privilégié de défense et d'attaque, mais aussi une « idole », un « totem » - termes employés ici dans le sens commun - le symbole de la bataille et de la lutte virile. En tant que tel, il est devenu rapidement le protagoniste par excellence des chants des moran.

3 Pour les Samburu le G-3 est vert

4 Encore le G-3. 


\section{Conclusion}

Les divisions intrinsèques au domaine musical entre moran et anciens recouvrent des aspects cruciaux pour la compréhension de l'idée d'identité individuelle et de groupe dans la société des Samburu. Si la définition du « soi » social se produit par le truchement de répertoires et de pratiques musicales à haute valeur représentative - qui à la fois séparent et unissent les composants des mêmes générations et des mêmes groupes d'âge - il faut s'interroger sur les conséquences de la disparition en cours de ces modes d'interaction au niveau musical sur la longue période. Le cas des Samburu du mont Nyiro offre un exemple éclairant sur la manière dont des bouleversements dans des modes de vie traditionnels peuvent avoir des effets immédiats non seulement sur les structures au fondement de la société, mais aussi sur un produit direct de ces structures et de cette société, telle que la musique. Il interroge en retour l'effet structurant de la performance musicale. Le nouveau visage d'une vieille guerre tribale a poussé les membres des communautés impliquées vers des formes d'alliance inconnues, qui ont aujourd'hui, comme dans le passé, besoin d'être traduites en musique pour préserver leur force et pour survivre.

\section{Bibliographie}

Mkutu, Kennedy A. Guns \& Governance in the Rift Valley. Pastoral Conflict \& Small Arms. Oxford: James Currey, 2008.

Pike, Ivy L., Bilinda Straight, et al. "Documenting the Health Consequences of Endemic Warfare in three Pastoralist Communities of Northern Kenya: A Conceptual Framework." Social Science \& Medicine 70, no. 1 (2010): 45-52.

Spencer, Paul. The Samburu. A Study of Gerontocracy in a Nomadic Tribe. London: Routledge \& Keagan Limited, 1965.

Spencer, Paul. Nomads in Alliance. Symbiosis and Growth among the Rendille and Samburu of Kenya. London: Oxford University Press, 1973.

Straight, Bilinda. "Cutting Time: Beads, Sex and Songs in the Making of Samburu Memory." In The Qualities of Time: Temporal Dimensions of Social Form and Human Experience, ed. Wendy James and David Mills, 267-283. Oxford: Berg, 2005 


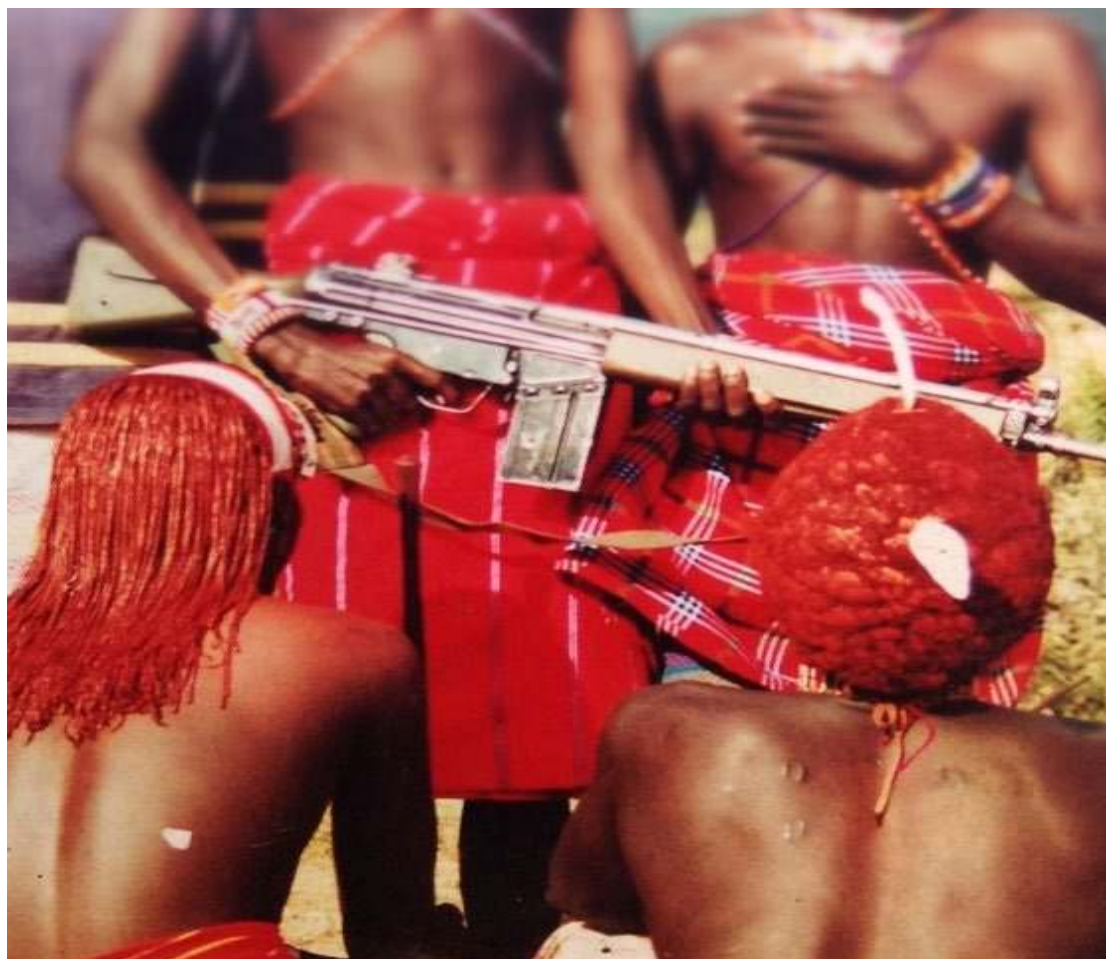

Présence de fusils d'assauts dès les années 1990 attestée par cette photographie d'archives 